\title{
A Regularity Result for CR Mappings Between Infinite Type Hypersurfaces
}

\author{
FRANCINE MEYLAN
}

Institut de Mathématiques, Université de Fribourg, Fribourg, Switzerland

\begin{abstract}
The Schwarz reflection principle in one complex variable can be stated as follows. Let $M$ and $M^{\prime}$ be two real analytic curves in $\mathbb{C}$ and $f$ a holomorphic function defined on one side of $M$, extending continuously through $M$, and mapping $M$ into $M^{\prime}$. Then $f$ has a holomorphic extension across $M$. In this paper, we extend this classical theorem to higher complex dimensions for a class of hypersurfaces of infinite type.
\end{abstract}

Keywords CR mappings; Infinite type; Real analytic hypersurfaces.

Mathematics Subject Classification $32 \mathrm{C} 16 ; 32 \mathrm{H} 02 ; 32 \mathrm{~V} 25$.

\section{Introduction}

Let $M$ be a (germ of a) Levi non-flat real analytic hypersurface at $p$ in $\mathbb{C}^{n}$. After a holomorphic change of coordinates, we may assume that $p=0$ and that there exists a sufficiently small open neighborhood $\Omega$ of 0 in $\mathbb{C}^{n}$ such that $M$ is given by an equation of the following form

$$
\operatorname{Im} w=(\operatorname{Re} w)^{m} \varphi(z, \bar{z}, \operatorname{Re} w), \quad(z, w) \in \mathbb{C}^{n-1} \times \mathbb{C}
$$

where $\varphi$ is a real valued convergent power series in $z, \bar{z}, \operatorname{Re} w$, such that

$$
\varphi(z, 0, \operatorname{Re} w) \equiv \varphi(0, \bar{z}, \operatorname{Re} w) \equiv 0, \quad \varphi(z, \bar{z}, 0) \not \equiv 0, \quad m \in \mathbb{N}
$$

Such a choice of coordinates is called normal coordinates. (See [1] for the existence of such coordinates). In this paper, we shall assume that $M$ is of infinite type (i.e., not of finite type in the sense of Bloom and Graham [6]) at 0 , which is equivalent to saying that $M$ contains a (germ of a) holomorphic hypersurface $S \subset M$ through 0 . Note that $M$ is of infinite type at 0 if and only if $m>0$. It is shown in [17] that the integer $m$ is a holomorphic invariant for normal coordinates. We have the following definition.

Address correspondence to Francine Meylan, Institut de Mathématiques, Université de Fribourg, 1700 Perolles, Fribourg, Switzerland; E-mail: francine.meylan@unifr.ch 
Definition 0.1. Let $M$ be of infinite type at 0 given by (0.1) and (0.2). We say that $M$ is $m$-nondegenerate at 0 if

$$
\operatorname{det}\left(\frac{\partial^{2} \varphi}{\partial z_{j} \partial \bar{z}_{k}}(z, \bar{z}, 0)\right) \not \equiv 0 .
$$

Note that if $M$ is $m$-nondegenerate at 0 , then $l(M)=1$, where $l(M)$ is the Levi type of $M$ in the sense of [3]. We shall see (Proposition 1.2) that the above definition is independent of the choice of normal coordinates. It should be noted here that normal coordinates are not unique.

The main result of this paper is the following.

Theorem 0.2. Let $M$ and $M^{\prime}$ be (germ of) real analytic hypersurfaces of infinite type at 0 in $\mathbb{C}^{n}$ given by $(0.1)$ and $(0.2)$. Suppose that $M$ (respectively $\left.M^{\prime}\right)$ is $m$-nondegenerate at 0 (respectively $m^{\prime}$-nondegenerate at 0 ). Let $D \subset \mathbb{C}^{n}$ be a domain with $M$ in its boundary. Let $f: M \rightarrow M^{\prime}$ be a continuous mapping which is the restriction of a certain continuous mapping over $\bar{D}$, holomorphic in $D$, with $f(0)=0$. Assume that $f$, as a map from $M$ into $M^{\prime}$, is finite to one. Then $f$ extends holomorphically to a neighborhood of 0 .

Observe that in the case where $n=2, M$ is $m$-nondegenerate at 0 if and only if $M$ is of infinite type at 0 and Levi non-flat. The following example shows that Theorem 0.2 fails if $M$ and $M^{\prime}$ are Levi flat.

Example 0.3. Let

$$
\begin{aligned}
M & =\left\{(z, w) \in \mathbb{C}^{2}: \operatorname{Im} w=0\right\} \\
M^{\prime} & =\left\{\left(z^{\prime}, w^{\prime}\right) \in \mathbb{C}^{2}: \operatorname{Im} w^{\prime}=0\right\}
\end{aligned}
$$

Consider

$$
f(z, w)=(z+h(z, w), w)
$$

where $h$ is a holomorphic function defined in the upper half plane in $\mathbb{C}$, smooth up to the boundary, but which does not extend holomorphically across 0 , with $h(0)=$ $h^{\prime}(0)=0$. Then one can check that $f$ is a local diffeomorphism near 0 , which maps $M$ into $M^{\prime}$. Obviously $f$ does not extend holomorphically at 0 .

The following example given in [15] shows that Theorem 0.2 may fail if we do not assume that $f$ is finite to one as a map from $M$ into $M^{\prime}$.

Example 0.4 [15]. Let

$$
\begin{aligned}
& M_{k}=\left\{(z, w) \in \mathbb{C}^{2}: \operatorname{Im} w=(\operatorname{Re} w)^{2}|w|^{2 k^{2}+k}|z|^{2 k}\right\}, \\
& M_{k}^{\prime}=\left\{\left(z^{\prime}, w^{\prime}\right) \in \mathbb{C}^{2}: \operatorname{Im} w^{\prime}=\left(\operatorname{Re} w^{\prime}\right)^{2}\left|z^{\prime}\right|^{2 k}\right\} .
\end{aligned}
$$

Consider

$$
f_{k}(z, w)=\left(z w^{k+\frac{1}{2}}, w\right),
$$


where $k$ is even. $M$ and $M^{\prime}$ satisfy the assumptions of Theorem 0.2 . One can check that $f_{k}$ is of class $C^{k, \frac{1}{2}}$, extends holomorphically to one side, maps $M_{k}$ into $M_{k}^{\prime}$, but is not of class $C^{k+1}$. Notice that $f_{k}$, as a map from $M_{k}$ to $M_{k}^{\prime}$ is not finite to one.

We would like to mention that the map $f$ given in the above example is $l$-tangentially finite in the sense of [17]. This condition is however sufficient to guarantee the holomorphic extendibility in the case where the map is assumed to be infinitely smooth, as shown in [17].

Finally, Theorem 0.2 also fails if $f$ does not extend to any side of $M$ as it is shown by the following example given in [9].

Example 0.5 ([9]). Let

$$
\begin{aligned}
M & =\left\{(z, w) \in \mathbb{C}^{2}: \operatorname{Im} w=(\operatorname{Re} w)|z|^{2}\right\}, \\
M_{k}^{\prime} & =\left\{\left(z^{\prime}, w^{\prime}\right) \in \mathbb{C}^{2}: \operatorname{Im} w^{\prime}=\left(\operatorname{Re} w^{\prime}\right) h_{k}\left(z^{\prime}, \bar{z}^{\prime}\right)\right\},
\end{aligned}
$$

where $h_{k}$ is defined as follows. Define the finite sequences $\left\{a_{k}\right\}$ and $\left\{b_{k}\right\}$ by

$$
\operatorname{Re}\left(w^{k}\right)=(\operatorname{Re} w)^{k}+\sum_{j=0}^{k-1} a_{j}(\operatorname{Re} w)^{j}(\operatorname{Im} w)^{k-j}, \quad \operatorname{Im}\left(w^{k}\right)=\sum_{j=0}^{k-1} b_{j}(\operatorname{Re} w)^{j}(\operatorname{Im} w)^{k-j},
$$

and put

$$
h_{k}=\frac{\sum_{j=0}^{k-1} b_{j}|z|^{2(k-j)}}{1+\sum_{j=0}^{k-1} a_{j}|z|^{2(k-j)}}
$$

Consider

$$
f_{k}(z, w)=\left(z, g_{k}(z, w)\right)
$$

where $g_{k}(z, w)=-w^{k}$ for $\operatorname{Re} w \leq 0$, and $g_{k}(z, w)=w^{k}$ for $\operatorname{Re} w>0$.

The reader can check that $f_{k}$ is a finite to one $C R$ map of class $C^{k-1}$, sending $M$ into $M_{k}^{\prime}$, which does not extend holomorphically to any neighborhood of 0 . Notice that $f_{k}$ is not the boundary value of any holomorphic map on $M$.

As in [15], we obtain the following result.

Theorem 0.6. Let $M, M^{\prime}$, and $f$ satisfy the assumptions of Theorem 0.2. Then $f=$ $\left(f_{1}, \ldots, f_{n-1}, g\right)$ extends as a locally proper holomorphic mapping from a neighborhood of 0 . Moreover there is an integer $l>0$ such that $g(z, w)=w^{l} g^{*}(z, w)$ with $g^{*}(0) \neq 0$.

We would like to mention that, under the assumptions of Theorem 0.6 , it does not mean that $f$ "preserves" the sides, as it is shown by the following example given in [15].

Example 0.7 ([15]). Let

$$
M=\left\{(z, w) \in \mathbb{C}^{2}: \operatorname{Im} w=(\operatorname{Re} w)|z|^{2}\right\},
$$




$$
M^{\prime}=\left\{\left(z^{\prime}, w^{\prime}\right) \in \mathbb{C}^{2}: \operatorname{Im} w^{\prime}=2\left(\operatorname{Re} w^{\prime}\right) \frac{\left|z^{\prime}\right|^{2}}{1-\left|z^{\prime}\right|^{4}}\right\}
$$

Consider

$$
f(z, w)=\left(z, w^{2}\right)
$$

One can check that $f$ maps $M$ into $M^{\prime}$. Notice that $f$ does not preserve the sides $(l=2)$.

In the complex plane the analogue of Theorem 0.2 is the Schwarz reflection principle, and there is no condition on the degeneracy of $M, M^{\prime}$, or $f$ at 0 , in contrast to higher dimensions as shown in Examples 0.3 and 0.4. We recall that the first reflection principles in higher dimensions were due to Lewy [16] and Pinchuk [18], and refer the interested reader to the book [1] and the survey article [14] for a precise account of the history. It should be noted that many results regarding the reflection principle require the map to be sufficiently smooth. In this paper, we only assume the map to be continuous. Theorem 0.2 is a generalization to higher dimensions of the results established in $\mathbb{C}^{2}$ for a continuous map by Ebenfelt and Huang [9]. It can also be seen as a generalization of the results given in the paper of Huang et al. [15]; indeed, if the map $f$ in Theorem 0.2 is assumed to be $C^{1}$, then Theorem 0.2 follows from the main theorem in [15].

We follow the same method as in [9] to prove Theorem 0.2.

In contrast to the $\mathbb{C}^{2}$ case, if $M$ is $m$-nondegenerate at 0 , it does not imply that we can find a point $p$ on the holomorphic hypersurface $S \subset M$ such that there exists a neighborhood $U$ of $p$ with $M$ strictly pseudoconvex at any point in $U \backslash S$. Therefore, in order to use the Pinchuk-Tsyganov theorem regarding the extendibility of CR continuous maps between strictly pseudoconvex domains [19], we first need to show that, under the assumptions of Theorem 0.2, pseudoconvex points are sent to pseudoconvex points (see Propositions 3.1 and 4.1).

We would like to mention that in the $\mathbb{C}^{2}$ case, using the work of Huang [12], Ebenfelt and Huang in [9] obtain Theorem 0.2, not only for hypersurfaces of infinite type, but more generally for Levi non-flat hypersurfaces.

\section{A Holomorphic Invariant}

In this section, we prove that Definition 0.1 is independent of the choice of normal coordinates.

Remark 1.1. If $M$ is of infinite type at 0 and given in normal coordinates by $(0.1)$ and (0.2), then $M$ contains (in the sense of germs) the holomorphic hypersurface $S$ given by

$$
S=\left\{(z, 0) \in \mathbb{C}^{n-1} \times \mathbb{C}\right\}
$$

Proposition 1.2. Definition 0.1 is independent of the choice of normal coordinates. 
Proof. Let $M^{\prime}$ be a real analytic hypersurface of infinite type at 0 , also given in normal coordinates by

$$
\operatorname{Im} w^{\prime}=\left(\operatorname{Re} w^{\prime}\right)^{m^{\prime}} \psi\left(z^{\prime}, \bar{z}^{\prime}, \operatorname{Re} w^{\prime}\right), \quad\left(z^{\prime}, w^{\prime}\right) \in \mathbb{C}^{n-1} \times \mathbb{C} .
$$

Let $H: M \rightarrow M^{\prime}$ be a biholomorphism between $M$ and $M^{\prime}$. Write $H=(F, G)$, where $F=\left(F_{1}, \ldots, F_{n-1}\right)$. Since $m$ is a holomorphic invariant, we have $m=m^{\prime}$. The proof of Proposition 2.11 in [17] shows that there exist a convergent power series $h(z, \bar{z})$ with $h(0) \neq 0$ such that

$$
h(z, \bar{z}) \varphi(z, \bar{z}, 0) \equiv \psi(F(z, 0), \bar{F}(\bar{z}, 0), 0) .
$$

First assume that

$$
\operatorname{det}\left(\frac{\partial^{2} \varphi}{\partial z_{j} \partial \bar{z}_{k}}(0,0,0)\right) \neq 0 .
$$

Using again the fact that we work in normal coordinates and that $h(0) \neq 0$, we conclude that Definition 0.1 is independent of the choice of normal coordinates. For the general case, we need the following lemmas.

Lemma 1.3. Let $M$ be a non Levi-flat real analytic hypersurface at 0 in $\mathbb{C}^{n}$ given in normal coordinates by (0.1) and (0.2). Assume that $M$ is of infinite type at 0 . Let $S$ be given by (1.1). Then the Taylor expansion of $\operatorname{Im} w-(\operatorname{Re} w)^{m} \varphi(z, \bar{z}, \operatorname{Re} w)$ at a point $\left(z_{0}, 0\right) \in S \cap M$ is given by

$$
\operatorname{Im} w-(\operatorname{Re} w)^{m} \tau\left(z-z_{0}, \bar{z}-\bar{z}_{0}, \operatorname{Re} w\right)
$$

where $\tau\left(z-z_{0}, \bar{z}-\bar{z}_{0}, \operatorname{Re} w\right)$ is a real valued convergent power series in $z-z_{0}$, $\bar{z}-\bar{z}_{0}, \operatorname{Re} w$, with $\tau\left(z-z_{0}, \bar{z}-\bar{z}_{0}, 0\right) \not \equiv 0$.

The easy proof of Lemma 1.3 is left to the reader.

Lemma 1.4. Let $M$ be a non Levi-flat real analytic hypersurface at 0 in $\mathbb{C}^{n}$ given in normal coordinates by (0.1) and (0.2). Assume that $M$ is of infinite type at 0 and $m$-nondegenerate. Let $S$ be given by (1.1). Then there exists a proper real analytic set $S_{0} \subset S$ such that for every $p \in S \backslash S_{0}$ there exist normal coordinates $(z, w)$ vanishing at $p$, in which $M$ can be defined, near $p=(0,0)$, by

$$
\operatorname{Im} w=(\operatorname{Re} w)^{m} \varphi(z, \bar{z}, \operatorname{Re} w), \quad(z, w) \in \mathbb{C}^{n-1} \times \mathbb{C},
$$

where $\varphi$ is a real valued convergent power series in $z, \bar{z}$, Re $w$, such that

$$
\operatorname{det}\left(\frac{\partial^{2} \varphi}{\partial z_{j} \partial \bar{z}_{k}}(0,0,0)\right) \neq 0 .
$$

Proof. After a linear holomorphic change of coordinates, we may assume, using Lemma 1.3 , that $M$ is given near 0 by

$$
\operatorname{Im} \tilde{w}=(\operatorname{Re} \tilde{w})^{m} \tilde{\varphi}(\tilde{z}, \overline{\tilde{z}}, \operatorname{Re} \tilde{w}), \quad(\tilde{z}, \tilde{w}) \in \mathbb{C}^{n-1} \times \mathbb{C}
$$


where $\tilde{\varphi}$ is a real valued convergent power series in $\tilde{z}, \overline{\tilde{z}}$, $\operatorname{Re} \tilde{w}$, with $\tilde{\varphi}(0)=0$, and

$$
\operatorname{det}\left(\frac{\partial^{2} \tilde{\varphi}}{\partial \tilde{z}_{j} \partial \tilde{\tilde{z}}_{k}}(0,0,0)\right) \neq 0 .
$$

Following the proof of Theorem 4.2.6 in [1], we may find normal coordinates $(z, w)$ satisfying $\tilde{z}=z, \tilde{w}=w+h(z, w)$, with $h$ holomorphic near the origin, $h(0)=0$ and $d h(0)=0$. One can check that in these coordinates, $M$ has the required properties. This proves the lemma.

We return to the proof of Proposition 1.2. After moving to a point $p \in M$ arbitrarily close to 0 , we can assume, following the proof of Lemma 1.4, that $M$ and $M^{\prime}$ are given in normal coordinates near 0 , such that (1.4) holds for $M$. The proof of the proposition then follows from the arguments at the beginning of the proof.

\section{A CR Extension Result}

In this section, we prove a CR extension theorem for $m$-nondegenerate hypersurfaces which are strictly pseudoconvex at any point in a neighborhood of 0 outside $S$.

Proposition 2.1. Let $M$ be given by (0.1) and (0.2) with $m>0, \varphi(z, \bar{z}, \operatorname{Re} w)=$ $\left|z_{1}\right|^{2}+\sum_{j=2}^{n-1} c_{j}\left|z_{j}\right|^{2}+O(3), c_{j} \in \mathbb{R}$, and let $h: M \rightarrow \mathbb{C}$ be a continuous $C R$ function. Then there exist positive numbers $\delta$ and $\epsilon$ such that $h$ extends holomorphically to the open set

$$
U_{\delta, \epsilon}^{++}=\left\{(z, w+i \tau) \in \Omega|(z, w) \in M,| z \mid<\epsilon, 0<\operatorname{Re} w<\epsilon, 0<\tau<\delta(\operatorname{Re} w)^{m}\right\} .
$$

Proof. Let

$$
M=\left\{(z, w) \in \Omega \mid \operatorname{Im} w=(\operatorname{Re} w)^{m} \varphi(z, \bar{z}, \operatorname{Re} w),(z, w) \in \mathbb{C}^{n-1} \times \mathbb{C}\right\},
$$

and

$$
\widetilde{M}=\left\{(z, w) \in \Omega \mid \operatorname{Im} w=\varphi(z, \bar{z}, \operatorname{Re} w),(z, w) \in \mathbb{C}^{n-1} \times \mathbb{C}\right\}
$$

Let

$$
\widetilde{\Omega}^{+}=\{(z, w) \in \Omega \mid \operatorname{Im} w>\varphi(z, \bar{z}, \operatorname{Re} w)\} .
$$

We put $w=s+i t$. Following the proof of Lewy's CR extension Theorem for hypersurfaces given in Section 15.2 of [5], we see that there exists an open set $V \in \mathbb{C}^{n-1}$, with $\bar{V} \supset\left\{\left(z_{2}, \ldots, z_{n-1}, s\right) \in \mathbb{C}^{n-1}:\left|z_{2}\right|<\epsilon_{0}, \ldots,\left|z_{n-1}\right|<\epsilon_{0},|s|<\epsilon_{0}\right\}$ for some $\epsilon_{0}>0$, such that if $\left(z_{2}, \ldots, z_{n-1}, s+i t\right) \in V$, then the complex line

$$
L_{z_{2}, \ldots, z_{n}, s+i t}=\left\{\left(\zeta, z_{2}, \ldots, z_{n}, s+i t\right) ; \zeta \in \mathbb{C}\right\}
$$

intersects $\widetilde{\Omega}^{+}$in a simply connected open subset of $L_{z_{2}, \ldots, z_{n}, s+i t}$ which we call $A_{z_{2}, \ldots, z_{n}, s+i t}$ whose boundary lies in $M$. Note that, by the Riemann mapping theorem, 
the $A_{z_{2}, \ldots, z_{n}, s+i t}$ are analytic discs attached to $M$ whose union clearly contains an open subset $U$ of $\widetilde{\Omega}^{+}$whose boundary contains a neighborhood of 0 in $M$. Let $\kappa: \mathbb{C}^{n} \longrightarrow$ $\mathbb{C}^{n}$ be given by $\kappa(z, s+i t)=\left(z, s+i\left(s^{m}\right) t\right)$. The map $\kappa$ sends $\widetilde{M}$ to $M$, and the open set $\kappa(U \cap\{s>0\})$ is filled by the simply connected open sets $A_{z_{2}, \ldots, z_{n}, s+i s^{m} t}$ whose boundary lies in $M \cap\{s>0\}$. Again, by the Riemann mapping theorem, we get the holomorphic extension in the open set given by (2.1) by standard arguments. (See the proof of Lewy's CR extension Theorem for hypersurfaces [5]). This achieves the proof of Proposition 2.1.

Remark 2.2. Following the proof of Proposition 2.1, we obtain open sets $U_{\delta, \epsilon}^{( \pm \pm)}$ defined in the same way as $U_{\delta, \epsilon}^{++}$, in which $f$ extends holomorphically in the cases where $\varphi(z, \bar{z}, \operatorname{Re} w)= \pm\left|z_{1}\right|^{2}+\sum_{j=2}^{n-1} c_{j}\left|z_{j}\right|^{2}+O(3), c_{j} \in \mathbb{R}$.

Using Proposition 2.1 and Remark 2.2, we obtain the following corollary.

Corollary 2.3. Let $M$ be given by (0.1) and (0.2) with $m>0, \varphi(z, \bar{z}, \operatorname{Re} w)=\left|z_{1}\right|^{2}-$ $\left|z_{2}\right|^{2}+\sum_{j=3}^{n-1} c_{j}\left|z_{j}\right|^{2}+O(3), c_{j} \in \mathbb{R}$, and let $h: M \rightarrow \mathbb{C}$ be a continuous CR function. Then there exist positive numbers $\delta$ and $\epsilon$ such that $h$ extends holomorphically to the open set

$$
U_{\delta, \epsilon}=\left\{(z, w+i \tau) \in \Omega|(z, w) \in M,| z|<\epsilon,| \operatorname{Re} w|<\epsilon,| \tau \mid<\delta(\operatorname{Re} w)^{m}\right\} .
$$

Remark 2.4. We would like to mention that if $M$ is given by an equation of the form

$$
\operatorname{Im} w=(\operatorname{Re} w)^{m} \varphi(z, \bar{z}, \operatorname{Re} w), \quad(z, w) \in \mathbb{C}^{n-1} \times \mathbb{C}
$$

where $\varphi(z, \bar{z}, \operatorname{Re} w)=\left|z_{1}\right|^{2}+\sum_{j=2}^{n-1} c_{j}\left|z_{j}\right|^{2}+O(3), c_{j} \in \mathbb{R}$, and $\varphi$ is of class $C^{3}$, then Proposition 2.1 still holds, using the same arguments.

\section{Image of a Pseudoconvex Point}

In this section, we show that if $f: M \rightarrow M^{\prime}$ is a continuous map which is the boundary value of a holomorphic map defined on one side of $M$, then pseudoconvex points are sent to pseudoconvex points provided $f$ is finite to one.

Proposition 3.1. Let $M$ and $M^{\prime}$ be real analytic hypersurfaces in $\mathbb{C}^{n}$, and $D \subset \mathbb{C}^{n}$ be a pseudoconvex domain with $M$ in its boundary. Let $f: M \rightarrow M^{\prime}$ be a continuous mapping which is the restriction of a certain continuous mapping over $\bar{D}$, holomorphic in $D$. Assume that $f$, as a map from $M$ into $M^{\prime}$, is finite to one. Then for any $p \in M, M^{\prime}$ is pseudoconvex at $f(p)$, and $\operatorname{Jac} f \not \equiv 0$ over $D$. of [2].

Before giving the proof of Proposition 3.1, we recall the following propositions

Proposition 3.2 ([2]). Let $M$ be a connected smooth hypersurface with $M \subset \partial \mathscr{G}$, where $\mathscr{G}$ is an open bounded domain in $\mathbb{C}^{n}$, and let $H$ be a holomorphic mapping in $\mathscr{O}$, 
continuous up to the boundary, $\mathrm{Jac} H \not \equiv 0$, with $H(M)$ contained in another smooth hypersurface $M^{\prime}$. Suppose $0 \in M, H(0)=0$, and

$$
H^{-1}(0) \cap \partial \odot \subset M
$$

Then there is a subdomain $\mathscr{O}_{1} \subset \mathcal{O}$ satisfying

(1) $0 \in \partial \mathscr{O}_{1}$, and there exists a sequence $\left\{z_{j}\right\} \subset \mathscr{O}_{1}$ such that $z_{j} \rightarrow 0$ and $H\left(z_{j}\right)$ stays strictly on one side of $M^{\prime}$.

(2) there exists $U$, a neighborhood of 0 in $M^{\prime}$, with $\overline{H\left(\mathscr{O}_{1}\right)} \supset U$;

(3) $H: \mathscr{O}_{1} \longrightarrow H\left(\mathscr{O}_{1}\right)$ is a proper map.

Proposition 3.3 ([2]). Let $\Omega$ and $\Omega^{\prime}$ be two bounded domains in $\mathbb{C}^{n}$ and $H$ a proper holomorphic mapping from $\Omega$ to $\Omega^{\prime}$. Suppose that $p_{0}$ and $p_{0}^{\prime}$ are boundary points of $\Omega$ and $\Omega^{\prime}$, respectively, and that there is a sequence $\left\{z_{j}\right\}_{j=1}^{\infty} \subset \Omega$ converging to $p_{0}$ such that $\lim _{j} H\left(z_{j}\right)=p_{0}^{\prime}$. Suppose that any holomorphic function in $\Omega^{\prime}$ is bounded on the sequence $\left\{H\left(z_{j}\right)\right\}_{j=1}^{\infty}$. Then $p_{0}$ is in the holomorphic hull of $\Omega$.

Proof of Proposition 3.1. Assume that $p=0$ and $f(0)=0$. By shrinking $M$ if necessary, we may assume that $f^{-1}(0) \cap M=\{0\}$. Suppose that there exists $E \subset$ $D$, irreducible component of $f^{-1}(0) \cap D$ which accumulates at 0 . We claim that $E$ has 0 dimension. Suppose by contradiction that $\operatorname{dim} E>0$. By a theorem of Remmert and Stein [7], $E \cup\{0\}$ is analytic near 0 . Since $D$ is pseudoconvex, using a theorem in [8], we may find a defining function $\rho$ for $M$ near 0 such that $-(-\rho)^{\alpha}$ is plurisubharmonic and $-(-\rho)^{\alpha}<0$ in $D$. Using the maximum principle [7], we reach a contradiction. The claim is then proved. In particular, this shows that if an irreducible component $A$ of $f^{-1}(q) \cap D, q \in M^{\prime}$, has strictly positive dimension, then

$$
\bar{A} \cap M=\emptyset .
$$

We claim that, shrinking $D$ if necessary, $f$, as a map from $\bar{D}$ to $f(\bar{D})$, is locally finite to one. Suppose not. Then there exist $p_{j} \in D$, with $p_{j} \rightarrow 0$, such that $\operatorname{dim} f^{-1}\left(f\left(p_{j}\right)\right)>0$. By (3.1), we can find $\lambda_{j k} \in \partial B_{k} \cap f^{-1}\left(f\left(p_{j}\right)\right) \cap D$, where $B_{k}$ is the ball centered at 0 of radius $k, 0<k<R$, for some $R>0$. Let $\lambda_{k} \in \partial B_{k}$ be an accumulation point of the sequence $\lambda_{j k}$. Since $f\left(\lambda_{j k}\right)=f\left(p_{j}\right)$, we conclude that $f\left(\lambda_{k}\right)=0$, for every $k$. Using the first part of the proof and the fact that $f$ is finite to one from $M$ into $M^{\prime}$, we get a contradiction. The map $f$ is then locally finite to one, and therefore

$$
\operatorname{Jac} f \not \equiv 0 \text {. }
$$

By the above, shrinking $D$ if necessary, we may assume that

$$
f^{-1}(0) \cap \partial D=\{0\}
$$

Using (3.2), (3.3) and Proposition 3.2, we conclude that there exist two domains $\Omega$ and $\Omega^{\prime}=f(\Omega)$ such that 0 is a boundary point for $\Omega$ and $\Omega^{\prime}, \Omega^{\prime}$ stays on one side of $M^{\prime}, \partial \Omega^{\prime}$ contains an open piece of $M^{\prime}$ at 0 , and $f$ is a proper map from $\Omega$ to $\Omega^{\prime}$. Suppose now that $M^{\prime}$ is not pseudoconvex at 0 . Then, by Proposition 3.3, we obtain 
that 0 is in the holomorphic hull of $\Omega$, and therefore $M$ is not pseudoconvex at 0 . This achieves the proof of Proposition 3.1.

\section{A Key Lemma}

In this section, following [9], we prove a lemma which establishes that the derivative of the map $f: M \rightarrow M^{\prime}$ along certain vector fields is continuous. This will allow us to apply certain vector fields to the equation expressing the fact that $f$ sends $M$ into $M^{\prime}$. (See (6.2) and (6.4)).

Proposition 4.1. Let $M$ and $M^{\prime}$ be real analytic hypersurfaces of infinite type at 0 in $\mathbb{C}^{n}$, satisfying (1.6) and (1.7). Let $D \subset \mathbb{C}^{n}$ be a domain with $M$ in its boundary. Let $f: M \rightarrow M^{\prime}$ be a continuous mapping which is the restriction of a certain continuous mapping over $\bar{D}$, holomorphic in $D$, with $f(0)=0$. Assume that $f$, as a map from $M$ into $M^{\prime}$, is finite to one. Then there exist positive numbers $\tilde{\delta}$ and $\tilde{\epsilon}$ such that $f$ extends to a bounded holomorphic function in the open set

$$
U_{\tilde{\delta}, \tilde{\epsilon}}=\left\{(z, w+i \tau) \in \Omega|(z, w) \in M,| z|<\tilde{\epsilon},| \operatorname{Re} w|<\tilde{\epsilon},| \tau \mid<\tilde{\delta}(|\operatorname{Re} w|)^{m}\right\}
$$

Before proving Proposition 4.1, we recall some known facts and notations.

There exist two open neighborhoods of $0, \Omega_{1}$ and $\Omega_{2}$, with $\Omega_{1} \subset \Omega_{2} \subset \Omega$, such that for every $p=\left(p^{\prime}, p_{n}\right) \in \Omega_{1}$, the Segre variety $Q_{p}$ of $M$ associated to $p$ is defined by

$$
Q_{p}=\left\{(z, w) \in \Omega_{2} \mid \frac{w-\overline{p_{n}}}{2 i}=\left(\frac{w+\overline{p_{n}}}{2}\right)^{m} \varphi\left(z, \overline{p^{\prime}}, \frac{w+\overline{p_{n}}}{2}\right)\right\} .
$$

It is shown in [10] that one can choose two sufficiently small balls $P$ and $\widetilde{P}$ centered at 0 such that the following holds

(i) $P \subset \subset \widetilde{P} \subset \Omega$;

(ii) There is a side-reversing (with respect to $M$ ) diffeomorphic map from $P$ into its image which is contained in $\widetilde{P}$, called the conjugate map $R$, such that $R(p) \in$ $Q_{p}$, for each $p \in P$. More precisely, that means that $R_{\mid M}=\mathrm{id}, R(p)$ and $p$ stay on different sides of $M$ for $p \notin M$.

Remark 4.2. The same notions are defined for $M^{\prime}$, that is, $\Omega^{\prime}, Q_{q}^{\prime}, P^{\prime}$ and $\widetilde{P}^{\prime}, R^{\prime}$.

We have the following lemma whose easy proof is left to the reader.

Lemma 4.3. Let $M$ be a real analytic hypersurface of infinite type at 0 , satisfying (1.6) and (1.7). Then

$$
Q_{p_{1}}=Q_{p_{2}} \Longleftrightarrow p_{1}=p_{2}
$$

provided $P$ is small enough and $p_{1}, p_{2} \in P \backslash S$. 
Proof of Proposition 4.1. Assume first that $\left(\frac{\partial^{2} \tilde{\varphi}}{\partial \tilde{z}_{j} \partial \tilde{z}_{k}}(0,0,0)\right)$ has eigenvalues of opposite signs. Using Corollary 2.3, we conclude that $f$ is the restriction of a holomorphic mapping on the open set

$$
U_{\delta, \epsilon}=\left\{(z, w+i \tau) \in \Omega|(z, w) \in M,| z|<\epsilon,| \operatorname{Re} w|<\epsilon,| \tau \mid<\delta(|\operatorname{Re} w|)^{m}\right\}
$$

for some choice of $\delta$ and $\epsilon$. Following the proof of Proposition 2.1, we have, by the maximum principle, that the sup of $f$ over $U_{\delta, \epsilon}$ is reached on $U_{\delta, \epsilon} \cap M$. This gives the desired conclusion.

Suppose now that $\left(\frac{\partial^{2} \tilde{\varphi}}{\partial \tilde{z}_{j} \partial \tilde{z}_{k}}(0,0,0)\right)$ has eigenvalues of same signs. We claim that

$$
f(M \backslash S) \cap S^{\prime}=\emptyset .
$$

Assume not. By Lemma 2.2 in [15], we conclude that the normal component of the map $f$ is identically 0 . This is a contradiction with Proposition 3.1. Using again Proposition 3.1 and a theorem of Pinchuk and Tsyganov [19], we conclude that $f$ extends holomorphically to a small neighborhood of $M \backslash S$. Without loss of generality, we assume that $M$ satisfies the assumptions of Proposition 2.1. We set

$$
U_{\delta, \epsilon}^{+-}=\left\{(z, w+i \tau) \in \Omega|(z, w) \in M,| z \mid<\epsilon, 0<\operatorname{Re} w<\epsilon,-\delta(\operatorname{Re} w)^{m}<\tau<0\right\} .
$$

We will always assume that $\widetilde{P}$ and $P^{\prime}$ are arranged such that

$$
f\left(\widetilde{P} \cap U_{\delta, \epsilon}^{++}\right) \cup R^{\prime}\left(f\left(\widetilde{P} \cap U_{\delta, \epsilon}^{++}\right)\right) \subset \subset P^{\prime}
$$

We define for $\tilde{\epsilon} \ll \epsilon, \tilde{\delta} \ll \delta$

$$
V=\left\{(p, q) \in\left(U_{\tilde{\delta}, \tilde{\epsilon}}^{+-} \cap P\right) \times P^{\prime} \mid f\left(Q_{p}^{c}\right) \subset Q_{q}^{\prime}\right\},
$$

where $Q_{p}^{c}$ is the connected component of $Q_{p} \cap U_{\tilde{\delta}, \tilde{\epsilon}}^{+}$containig $R(p)$. Let $\widetilde{V}$ be the irreducible component of $V$ which contains the graph of $f$, and let $\pi$ be the natural projection from $\widetilde{V}$ to $U_{\tilde{\delta}, \tilde{\epsilon}}^{+-} \cap P$. We need the following lemma whose proof is an adjustment of Lemma 3.1 in [12] to higher dimensions. For the convenience of the reader, we will give its proof.

Lemma 4.4. Let $\widetilde{V}$ and $\pi$ as above. Then $\pi$ is surjective.

Proof. Suppose that $\pi$ is not surjective. We observe that $\pi: \widetilde{V} \longrightarrow U_{\tilde{\delta}, \tilde{\epsilon}}^{+-} \cap P$ is an open mapping. Let $p \in \overline{U_{\tilde{\delta}, \tilde{\epsilon}}^{+-}} \cap P \cap M$. As in [12], we may then find a curve $\gamma:(0,1] \longrightarrow U_{\tilde{\delta}, \tilde{\epsilon}}^{+-} \cap P$ satisfying the following properties: $\gamma((0,1)) \subset \pi(\widetilde{V}), p=$ $\lim _{t \rightarrow 0^{+}} \gamma(t)$ and $\gamma(1) \notin \pi(\widetilde{V})$. Lifting $\gamma$ and using the fact that $\pi: \widetilde{V} \longrightarrow U_{\tilde{\delta}, \tilde{\epsilon}}^{+-} \cap P$ is locally proper, we can find a unique lift of $\gamma$ to $\tilde{\gamma}:[0,1] \longrightarrow \mathbb{C}^{n} \times \mathbb{C}^{n}$ with the following properties: $\tilde{\gamma}((0,1)) \subset \widetilde{V}, \tilde{\gamma}(0)=(p, f(p))$. We put $\tilde{\gamma}(t)=(\gamma(t), q(t))$ for $t \in(0,1)$. By definition of $\widetilde{V}$, we have $f\left(Q_{\gamma(t)}^{c}\right) \subset Q_{q(t)}^{\prime}$ for $t \in(0,1)$. By a theorem of Baouendi and Rothschild [4], we know that the local holomorphic extension of $f$ near $p$ is locally proper near $p$. It follows, using Lemma 2.1(f) in [13], that for each small $t, R^{\prime}(q(t)) \in f\left(Q_{\gamma(t)}^{c}\right)$. 
We claim that

$$
R^{\prime}(q(t)) \in f\left(Q_{\gamma(t)}^{c}\right) \text { for } t \in(0,1)
$$

Assuming the claim, we obtain that for each $t$, there exists $u(t) \in Q_{\gamma(t)}^{c}$ with

$$
f(u(t))=R^{\prime}(q(t)) .
$$

Using (4.6), we obtain that

$$
\{q(t), t \in(0,1)\} \subset \subset P^{\prime} .
$$

That means that all the limit points of $q(t)$ as $t \rightarrow 1^{-}$stay in $P^{\prime}$. Let $q(1)$ be one of these limit points. We then have $f\left(Q_{\gamma(1)}^{c}\right) \subset Q_{q(1)}^{\prime}$, which is a contradiction. To complete the proof of the lemma, we need to prove the claim (4.8). As in Lemma 3.1 in [12], we let $\delta=\max \left\{\tau \in(0,1] \mid\right.$ for $\left.t \in(0, \tau), R^{\prime}(q(t)) \in f\left(Q_{\gamma(t)}^{c}\right)\right\}$. We have $0<$ $\delta \leq 1$. Suppose $\delta<1$. Then there exists $\left.u(t) \in Q_{\gamma(t)}^{c}\right)$ with $f(u(t))=R^{\prime}(q(t))$, for each $t \in(0, \tau)$. Let $u(\delta)=\lim _{t_{j} \rightarrow \delta^{-}} u(t)$. We have $\left.u(\delta) \in Q_{\gamma(\delta)}^{c}\right)$. By definition, we have

$$
f\left(Q_{\gamma(\delta)}^{c}\right) \subset Q_{q(\delta)}^{\prime}
$$

We also get

$$
f(u(\delta))=R^{\prime}(q(\delta))
$$

Assume first that $u(\delta) \in M$. As $f(u(\delta))=R^{\prime}(q(\delta)) \in M^{\prime}$, we have $q(\delta) \in M^{\prime}$. Using (4.9) and applying Lemma 2.1(f) in [13], we then obtain a contradiction since $f$ is locally finite.

Suppose now that $u(\delta) \notin M$. Since $\delta<1$, there exists a sequence $t_{j} \rightarrow \delta^{+}$such that

$$
R^{\prime}\left(q\left(t_{j}\right)\right) \notin f\left(Q_{\gamma\left(t_{j}\right)}^{c}\right)
$$

Let $B_{\epsilon}(u(\delta))$ be the ball centered at $u(\delta)$ with radius $\epsilon$. Following the proof of the last part of Lemma 3.1 in [12] and using (4.11), for $t_{j}$ close to $\delta$ and $\epsilon$ sufficiently small, we may find a biholomorphic map $\varphi_{t_{j}}$, depending continuously on $t_{j}$, from the unit disk to $Q_{\gamma\left(t_{j}\right)}^{c} \cap B_{\epsilon}(u(\delta))$, such that the map $f \circ \varphi_{t_{j}}-R^{\prime}\left(q\left(t_{j}\right)\right)$ never vanishes on the unit disk. Noticing that the Hurwitz theorem is still valid in higher dimension in the case where $f$ is locally finite, we may then conclude that $f \circ \varphi_{\delta}-R^{\prime}(q(\delta))$ never vanishes on the unit disk. This contradicts (4.10). This achieves the proof of the claim, and therefore the proof of the lemma.

We now return to the proof of Proposition 4.1. Using (the proof of) Propositions 2.1, 3.1, (4.4), the maximum principle and the Hopf Lemma, we observe that if $p \in U_{\tilde{\delta}, \tilde{\epsilon}}^{+-} \cap P$, then $f\left(Q_{p}^{c}\right) \cap M^{\prime}=\emptyset$. In particular, this shows that if $(p, q) \in V$, then $q \notin S$. Hence, using Lemmas 4.3 and 4.4 , we may define the following holomorphic map.

$$
\begin{aligned}
\tilde{f}:\left(U_{\tilde{\delta}, \tilde{\epsilon}}^{+-} \cap P\right) & \longrightarrow P^{\prime} \\
p & \longrightarrow \pi^{\prime} \circ \pi^{-1}(p)
\end{aligned}
$$


where $\pi^{\prime}$ is the natural projection from $\widetilde{V}$ to $P^{\prime}$. This map $\tilde{f}$ gives the desired holomorphic extension of $f$ in $\left(U_{\tilde{\delta}, \tilde{\epsilon}}^{-} \cap P\right)$. Following the proof of Lemma 4.4, we notice that there exists $u \in Q_{p}^{c}$ such that

$$
R^{\prime}(\tilde{f}(p))=f(u)
$$

Therefore, using (4.13), (the proof of) Proposition 2.1, and the maximum principle, we conclude that $\tilde{f}$ is bounded in $\left(U_{\tilde{\delta}, \tilde{\epsilon}}^{+-} \cap P\right)$ by the sup of $f$ on $M$. This completes the proof of Proposition 4.1.

We write $w=s+i t$ and denote by $L_{j}, j=1, \ldots, n-1$, the antiholomorphic vector fields tangent to $M$ given by

$$
L_{j}=\frac{\partial}{\partial \bar{z}_{j}}-2 i \frac{s^{m} \varphi_{\bar{z}_{j}}}{1+i\left(s^{m} \varphi\right)_{s}} \frac{\partial}{\partial \bar{w}} .
$$

Note that they form a basis of the $C R$ vector fields on $M$. By the theorem of Pinchuk and Tsyganov [19], we know that $L_{j} f, j=1, \ldots, n$, is real analytic outside $S$. We have the following extension lemma for $L_{j} f, j=1, \ldots, n$.

Lemma 4.5. Let $M$ and $M^{\prime}$ be real analytic hypersurfaces of infinite type at 0 in $\mathbb{C}^{n}$, satisfying (1.6) and (1.7). Let $f: M \rightarrow M^{\prime}$ be a continuous mapping which is the restriction of a certain continuous mapping over $\bar{D}$, holomorphic in $D$, with $f(0)=0$. Assume that $f$, as a map from $M$ into $M^{\prime}$, is finite to one. Then $\overline{L_{j}} f, j=1, \ldots, n$, extends continuously to a neighborhood of 0 in $M$.

Proof. Let

$$
U_{\tilde{\delta}, \tilde{\epsilon}}=\left\{(z, w+i \tau) \in \Omega|(z, w) \in M,| z|<\tilde{\epsilon},| \operatorname{Re} w|<\tilde{\epsilon},| \tau \mid<\tilde{\delta}(|\operatorname{Re} w|)^{m}\right\}
$$

given by Proposition 4.1. For fixed $(z, w) \in M \backslash S$, we define $H_{k}$ to be the holomorphic function in $\eta,|\eta|<\tilde{\delta}|\operatorname{Re} w|^{m}$, given by $H_{k}(\eta)=f_{k}(z, w+\eta), \quad k=$ $1, \ldots, n$, where $f_{k}$ are the components of $f$. Using the Cauchy estimates for $H_{k}(\eta)$ at $\eta=0$ and Proposition 4.1, we get

$$
\left|\frac{\partial f_{k}}{\partial w}(z, w)\right| \leq \frac{C}{|\operatorname{Re} w|^{m}}
$$

where $C>0$ is a constant depending only on $\epsilon$.

Define the family of functions in $\zeta$, parametrized by $(z, w) \in M$, by

$$
\left\{K_{k(z, w)}(\zeta)=f_{k}(z+\zeta, w), k=1, \ldots, n\right\} .
$$

We observe that $\left\{K_{k(z, w)}\right\}$ forms a family of holomorphic functions in $\zeta$, for $|\zeta|$ small enough. Using the Cauchy estimates and Taylor's expansion, we observe that $K_{k(z, w)}(\zeta)$ converges to $f_{k}(\zeta, 0)$ uniformly on compact sets as $(z, w) \rightarrow 0$, for $k=$ $1, \ldots, n$. We therefore obtain that

$$
\lim _{(z, w) \rightarrow 0} \frac{\partial f_{k}}{\partial z_{j}}(z, w)=\frac{\partial f_{k}}{\partial z_{j}}(0,0) \quad k=1, \ldots, n, j=1, \ldots, n-1 .
$$


Let $\overline{L_{j}}$ defined by (4.14). Using (1.6), (4.16) and (4.18), we obtain that

$$
\lim _{(z, w) \rightarrow 0} \overline{L_{j}} f_{k}(z, w)=\frac{\partial f_{k}}{\partial z_{j}}(0,0) \quad k=1, \ldots, n, j=1, \ldots, n-1 .
$$

Using Lemma 1.4, for any point $p \in S$ close enough to 0 , we can choose normal coordinates vanishing at $p$ satisfying (1.6) and (1.7). In these new coordinates, (4.19) will be valid. Going back to the coordinates we started with, we get that $\lim _{(z, w) \rightarrow p} \bar{L}_{j} f_{k}(z, w), k=1, \ldots, n, j=1, \ldots, n-1$, exists, for every $p \in S$ close to 0 . Using (4.19) and the fact that the normal coordinates depend smoothly on the point $p$, we obtain that $\overline{L_{j}} f, j=1, \ldots, n-1$, extends continuously to a neighborhood of 0 in $M$. This achieves the proof of the lemma.

\section{Regularity Property of the Transversal Component}

Let $M$ and $M^{\prime}$ be real analytic hypersurfaces of infinite type at 0 in $\mathbb{C}^{n}$ given by $(0.1)$ and (0.2). Let $D \subset \mathbb{C}^{n}$ be a domain with $M$ in its boundary. Let $f: M \rightarrow M^{\prime}$ be a continuous mapping which is the restriction of a certain continuous mapping over $\bar{D}$, holomorphic in $D$, with $f(0)=0$. Assume that $f$, as a map from $M$ into $M^{\prime}$, is finite to one. We write $f=\left(f^{*}, g\right)=\left(f_{1}^{*}, \ldots, f_{n-1}^{*}, g\right)$. Recall that $g$ is denoted the transversal component of $f$. Using Proposition 4.1, we see that $g$ cannot be identically 0 . Let $S$ be defined by (1.1) and let $T \subset M$ be a totally real submanifold of real dimension $n$ passing through 0 such that $T \cap S$ is totally real of real dimension $n-1$ near 0 . We may assume, without loss of generality, that $T=\mathbb{R}^{n}$ near 0 . Notice that in these new coordinates, $M$ is in general not given in normal coordinates; nevertheless, we still call $g$ the transversal component.

For $\delta>0$ we define, as in [15], the following wedges with edge $T$

$$
\mathscr{W}_{\delta}^{ \pm}=\left\{(z, w) \mid \pm \operatorname{Im} w>\delta\left(\left|\operatorname{Im} z_{1}\right|+\cdots+\left|\operatorname{Im} z_{n-1}\right|\right)\right\} .
$$

For the rest of the section, we fix $\delta$ such that $\mathscr{W}_{\delta}^{+}$(respectively $\mathscr{W}_{\delta}^{-}$) lies "above" (respectively "below") $M$ in the sense of germs at the origin. Again, without loss of generality, we may assume that $\mathscr{W}_{\delta}^{+} \subset D$ in the sense of germs at the origin. Let

$$
\beta=\left(\beta_{1}, \ldots, \beta_{n}\right) \in \mathbb{R}^{n}, \quad \beta^{\prime}=\left(\beta_{1}^{\prime}, \ldots, \beta_{n-1}^{\prime}\right) \in \mathbb{R}^{n-1} .
$$

For $\epsilon>0$, we define

$$
\Delta_{\epsilon}=\{\zeta \in \mathbb{C}|| \zeta \mid<\epsilon\}, \quad \Delta_{\epsilon}^{+}=\{\zeta \in \mathbb{C} \mid \operatorname{Im} \zeta>0\} .
$$

For $\beta_{n}>\delta\left(\left|\beta_{1}\right|+\cdots+\left|\beta_{n-1}\right|\right.$ and for $\epsilon$ sufficiently small, we define $\psi_{\beta, \beta^{\prime}}$ by

$$
\zeta \longrightarrow\left(\beta_{1} \zeta+\beta_{1}^{\prime}, \ldots, \beta_{n-1} \zeta+\beta_{n-1}^{\prime}, \beta_{n} \zeta\right)
$$

We observe that $\psi_{\beta, \beta^{\prime}}\left(\Delta_{\epsilon}^{+}\right) \subset D$.

The following proposition is proved in [9] in the case $n=2$, but its proof is totally similar for the general case $n \geq 2$, using (5.2), (5.3), and (5.4). We then refer the reader to Proposition 3.1 and Lemma 3.2 in [9] for the details of the proof. 
Proposition 5.1. Assume that $M, M^{\prime}$ and $f$ satisfy the above. Then there exist a point $p=\left(\beta^{\prime}, 0\right) \in S \cap T$ near 0 , and an integer $N>0$ such that the transversal component $g$ satisfies

$$
|\overline{g(\bar{z}, \bar{w})}| \geq|\operatorname{lm} w|^{N}
$$

for $(z, w) \in \mathscr{W}_{\delta}^{-} \cap O(p)$, where $O(p)$ is a small neighborhood of $p$.

\section{Proof of Theorem 0.2}

In this section we give the proof of Theorem 0.2, using Lemma 4.5 and Proposition 5.1. Another ingredient of the proof is the following propagation theorem due to Hanges and Treves [11], which was also used in [9, 15].

Theorem 6.1. Let $M$ be as in Theorem 0.2. Let $h$ be a CR function over $M$. If for a certain $p \in S, h$ extends holomorphically to a neighborhood of $p$, then $h$ extends holomorphically to a neighborhood of $S$.

Proof of Theorem 0.2. Since $f$, as a map from $M$ to $M^{\prime}$ is finite to one, we conclude, using Proposition 4.1 and Lemma 2.2 in [15], that $f$ is a local biholomorphic map from $S$ to $S^{\prime}$ away from a thin set. Using Lemma 1.4, we may then assume that $M$ and $M^{\prime}$ satisfy the assumptions of Proposition 4.1 so that $f^{*}$ is a local biholomorphism at 0 . We may also assume that (5.5) holds at $p=0$. Let $M^{\prime}$ be given by (1.2). Since $f(M) \subset M^{\prime}$, we get that

$$
\operatorname{Im} g(z, w)=\operatorname{Re} g(z, w)^{m^{\prime}} \psi\left(f^{*}(z, w), \overline{f^{*}(z, w)}, \operatorname{Re} g(z, w)\right)
$$

for $(z, w) \in M$. Applying the implicit function theorem, we obtain the following functional equation

$$
\overline{g(z, w)}=g(z, w)+g(z, w)^{m^{\prime}} H\left(f^{*}(z, w), \overline{f^{*}(z, w)}, g(z, w)\right)
$$

for $(z, w) \in M$. Since $M^{\prime}$ is given in normal coordinates, it is easy to see that

$$
\operatorname{det}\left(\frac{\partial^{2} H}{\partial z_{j} \partial \bar{z}_{k}}(0,0,0)\right) \neq 0
$$

Applying $L_{j}$ defined by (4.14) (via the parametrization) to (6.2), we obtain

$$
L_{j} \overline{g(z, w)}=g(z, w)^{m^{\prime}} \sum_{k=1}^{n-1} H_{\bar{z}_{k}^{\prime}}\left(f^{*}(z, w), \overline{f^{*}(z, w)}, g(z, w)\right) L_{j} \overline{f_{k}^{*}(z, w)}
$$

Using Cramer's rule, we obtain that

$$
\operatorname{det}\left(L_{j} \overline{f_{k}^{*}(z, w)}\right) g(z, w)^{m^{\prime}} H_{\bar{z}_{k}^{\prime}}\left(f^{*}(z, w), \overline{f^{*}(z, w)}, g(z, w)\right)=h_{k},
$$


where $h_{k}$ extends holomorphically to $\mathscr{W}_{\delta}^{-}$and continuously up to $T$. Using (4.19) (which still holds after the change of coordinates for $M$ given in Section 5, and using the fact that $f^{*}$ is a local biholomorphism at 0 , we conclude that

$$
g(z, w)^{m^{\prime}} H_{z_{k}^{\prime}}\left(f^{*}(z, w), \overline{f^{*}(z, w)}, g(z, w)\right)=\frac{h_{k}}{\operatorname{det}\left(L_{j} \overline{f_{k}^{*}(z, w)}\right)} .
$$

Taking the conjugate of the equation defined by (6.2), and using (6.6), we obtain the following equation

$$
\begin{aligned}
& \left.\left(1+g(z, w)^{m^{\prime}-1} H\left(f^{*}(z, w)\right), \overline{f^{*}(z, w)}, g(z, w)\right)\right)^{m^{\prime}} H_{\bar{z}_{k}^{\prime}}\left(f^{*}(z, w), \overline{f^{*}(z, w)}, g(z, w)\right) \\
& =\frac{h_{k}}{\operatorname{det}\left(L_{j} \overline{f_{k}^{*}(z, w)}\right) \overline{g(z, w)} \bar{m}^{\prime}} .
\end{aligned}
$$

We see, using the Cauchy estimates, that $L_{j} \overline{f_{k}(z, w)}, k=1, \ldots, n$ extends as a holomorphic function of slow growth in the wedge $\mathscr{W}_{\delta}^{-}$in the sense of germs at the origin. Using Lemma 4.5, we conclude that $L_{j} \overline{f_{k}(z, w)}, k=1, \ldots, n$ extends holomorphically to $\mathscr{W}_{\delta}^{-}$and continuously up to $T$. Therefore, using Proposition 5.1, we obtain that the right hand side of (6.7) extends holomorphically to $\mathscr{W}_{\delta}^{-}$and continuously up to $T$.

Put

$$
R_{k}=\frac{h_{k}}{\operatorname{det}\left(L_{j} \overline{f_{k}^{*}(z, w)}\right) \overline{g(z, w)}} \bar{m}^{\prime}, \quad k=1, \ldots, n-1 .
$$

Using (6.3) as well as the implicit function theorem, we get, after taking the conjugate of (6.2) again

$$
f^{*}(z, w)=Y\left(\overline{f^{*}(z, w)}, \overline{g(z, w)}, R\right),
$$

where $Y$ is some holomorphic vector-valued function of its arguments, $R=$ $\left(R_{1}, \ldots, R_{n-1}\right)$, and $(z, w) \in T$. By the above discussion, using (6.9), we obtain that $f^{*}$ extends holomorphically to $\mathscr{W}_{\delta}^{-}$and continuously up to $T$. Therefore, taking again the complex conjugate of (6.2), we obtain that $g$ extends holomorphically to $\mathscr{W}_{\delta}^{-}$and continuously up to $T$. Using the classical edge of the wedge theorem, we get that $f$ extends holomorphically to an open neighborhood of 0 . By Theorem 6.1, $f$ extends holomorphically to a neighborhood of $S$. This achieves the proof of Theorem 0.2.

Proof of Theorem 0.6. By Theorem 0.2, we obtain in particular that $f$ is a $C^{1}$ mapping; therefore, we may apply the corollary of [15] to conclude. This achieves the proof of Theorem 0.6.

\section{Acknowledgments}

The author was partially supported by Swiss NSF Grant 2100-063464.00/1.

I would like to thank X. Huang and Nordine Mir for many helpful discussions and comments on this paper. I am also grateful to the referee for several useful remarks. 


\section{References}

[1] Baouendi, M. S., Ebenfelt, P., Rothschild, L. P. (1999). Real Submanifolds in Complex Space and Their Mappings. Princeton, NJ: Princeton University Press.

[2] Baouendi, M. S., Huang, X., Rothschild, L. P. (1995). Nonvanishing of the differential of holomorphic mappings at boundary points. Math. Res. Let. 2:737-750.

[3] Baouendi, M. S., Huang, X., Rothschild, L. P. (1996). Regularity of CR mappings between algebraic hypersurfaces. Invent. Math. 125:13-36.

[4] Baouendi, M. S., Rothschild, L. P. (1990). Geometric properties of mappings between hypersurfaces in complex space. J. Differential Geom. 31:473-499.

[5] Boggess, A. (1991). CR Manifolds and the Tangential Cauchy-Riemann Complex. Boca Raton, FL: CRC Press.

[6] Bloom, T., Graham, I. (1977). On type conditions for generic real submanifolds of $\mathbb{C}^{n}$. Invent. Math. 40:217-243.

[7] Chirka, E. M. (1989). Complex Analytic Sets. Dordrecht, The Netherlands: Kluwer Academic Publishers.

[8] Diederich, K., Fornaess, J. E. (1977). Pseudoconvex domains: Bounded strictly plurisubharmonic exhaustion functions. Invent. Math. 39:129-141.

[9] Ebenfelt, P., Huang, X. (2001). On a generalized reflection principle in $\mathbb{C}^{2}$. Complex Analysis and Geometry, Ohio State Univ. Math. Res. Inst. Publ. 9:125-140.

[10] Forstnerič, F. (1989). Extending proper holomorphic mappings of positive codimension. Invent. Math. 95:31-61.

[11] Hanges, N., Treves, F. (1983). Propagation of holomorphic extendibility of CR functions. Math. Ann. 263:157-177.

[12] Huang, X. (1996). Schwarz reflection principle in complex space of dimension two. Comm. Partial Differential Equations 21:1781-1828.

[13] Huang, X. (2000). A removable singularity property for CR mappings between real analytic hypersurfaces. Comm. Partial Differential Equations 25:299-317.

[14] Huang, X. (2001). On some problems in several complex variables and CR geometry. In: First International Congress of Chinese Mathematicians (Beijing, 1998), eds. L. Yang, S.-T. Yau. AMS/IP Stud. Adv. Math. Vol. 20, pp. 383-396. Providence, RI: American Mathematical Society.

[15] Huang, X., Merker, J., Meylan, F. (2000). Mappings between degenerate real analytic hypersurfaces in $\mathbb{C}^{n}$. In: Analysis, Geometry, Number Theory: The Mathematics of Leon Ehrenpreis, ed. E. I. Grinberg. Contemp. Math. Vol. 251, pp. 321-338. Providence, RI: American Mathematical Society.

[16] Lewy, H. (1977). On the boundary behavior of holomorphic mappings. Acad. Naz. Lincei 35:1-8.

[17] Meylan, F. (1995). A reflection principle in complex space for a class of hypersurfaces and mappings. Pacific Journal of Mathematics 169:135-160.

[18] Pinchuk, S. (1974). Bogoljubov's theorem on the "edge of the wedge" for generic manifolds. Math. USSR Sbornik 23:441-455.

[19] Pinchuk, S., Tsyganov, S. (1990). The smoothness of CR mappings between strictly pseudoconvex hypersurfaces. Math. USSR Izvestiya 35:457-467. 